\title{
IMPROVING GROUND THERMAL VACUUM TESTING FOR SMALL SATELLITES OF THE "AIST" FAMILY
}

\author{
S.I. Tkachenko, V.V. Salmin, I.S. Tkachenko, S.L. Safronov, \\ I.V. Kaurov, M.D. Korovin, M.A. Ivanushkin, S.S. Volgin \\ Samara National Research University, Samara, Russia
}

\begin{abstract}
The purpose of this study is to perform an analysis of the process of experimental testing of the thermal control system of the "AIST" small satellite, using the computer simulation of the satellite's flight in the "TERM" software package. Based on the telemetry data, obtained by the Samara University satellite ground control center, actualized parameters of the ground testing process were proposed. The ground testing parameters were verified by calculation of the satellite's panels temperatures using the "ANSYS Thermal" software system both for the initial conditions obtained from the ground testing documentation and the ones that were proposed after the analysis of the thermal flow on the satellite.
\end{abstract}

Keywords: thermal-vacuum testing, satellite, CAE, thermal control system, thermal flux.

Citation: Tkachenko SI, Salmin VV, Tkachenko IS, Safronov SL, Kaurov IV, Korovin MD, Ivanushkin MA, Volgin SS. Improving ground thermal vacuum testing for small satellites of the "AIST" family. CEUR Workshop Proceedings, 2016; 1638: 691-699. DOI: 10.18287/1613-0073-2016-1638-691-699

\section{Introduction}

Experimental testing of the satellite's thermal control system (TCS) is a technically challenging process. TCS must protect the satellite both from overheating and freezing. The thermal control of the satellite is further complicated by the fact that it performs an unoriented flight, which makes it impossible to know in advance, what panels of the satellite will receive the most heat.

The performance study of the thermo control system was carried out on "AIST" (RS43as) and "AIST" (RS-41at) small satellites. The "AIST" small satellites family was created as a result of a joint project between RSC "Progress" (Samara, Russia) and Samara State Aerospace university (SSAU). Small satellite "AIST" (RS-43as) was launched as a way cargo on the "BION-M" satellite No. 1 on 19th of April, 2013. "AIST" (RS-41as) was put into orbit with the "SOUYZ-2.1v" on 28th of December, 2013 from the Plesetsk cosmodrome. Scientific and educational goals of the "AIST" 
satellites are disclosed in [1]. The parameters of the satellite's orbits are listed in table 1.

Table 1. Orbital parameters of the „AIST“ satellites

\begin{tabular}{lll}
\hline & "AIST" (RS-43as) & "AIST" (RS-41at) \\
\hline Perigee, $\mathrm{km}$ & 569.8 & 595.1 \\
Apogee, $\mathrm{km}$ & 583.0 & 621.6 \\
Inclination, deg. & 64.9 & 82.4 \\
Period, min. & 96 & 97 \\
Semimajor axis, km & 6947 & 6986 \\
\hline
\end{tabular}

By comparing the results of the ground testing with the telemetry data, received during the active part of the mission it is possible to validate the ground testing procedures and to enhance them for future spacecraft of similar type. It would allow to create satellites with greater resource and therefore increase longevity of their missions.

\section{Problem setting}

\subsection{Ground testing}

Thermo-vacuum testing of the "AIST" satellites was carried out with the TVU 400-05 "RCS Progress's" testing chamber in 2012.

TCS serves to maintain the onboard temperature of the satellite within a certain range that allows the onboard equipment to function properly during the mission. The TCS of the "AIST" satellite consists of thermal regulation system and the passive means of thermal regulation. The passive means of thermal regulation include thermal pipes, thermal control coating, and thermal resistances. The thermal-vacuum testing included extreme conditions both in overcooling and overheating modes with regard to the external heat flux and the heat from the onboard equipment. The estimated deviation of the testing conditions was $\pm 10 \%$. Testing was conducted in a vacuum chamber under pressure of not more than $1 \times 10^{-5} \mathrm{~mm}$. Hg. Thermal influence of space was emulated with screens that had the emissivity factor of more than 0.9 and were cooled with liquid nitrogen to the temperature of minus $180( \pm 10){ }^{\circ} \mathrm{C}$. Since the "AIST" telemetry data indicates that the satellites are almost always overheated, this study only considers the overheating mode. During the simulation in the "TERM" software package, maximum heat flux were applied, revolution duration - 90 minutes, no shadows. The plane of the panel "- X" is aimed at the Earth (Figure 1).

\subsection{Ground control center}

Ground control center (GCC) receives the telemetry from the moment of the launch of the first "AIST" satellite. GCC performs the task of ground flight control of the "AIST" satellites (Figure 2). The main mode of "AIST" satellites - standalone using the principles of operational management in the areas of radio coverage. The autono- 
mous functioning program is formed by the GCC and is recorded in the onboard memory of the satellite during the communication session.

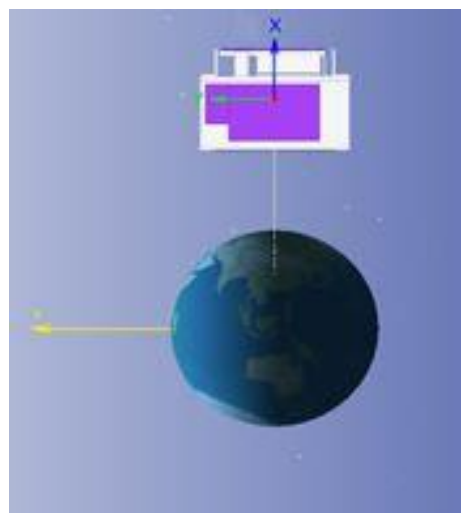

Fig. 1. Orientation of the satellite in the overheating mode

As for 20th of March 2016 the first "AIST" (RS-43as) small satellite made 15129 circulations around the Earth, 7256 communication sessions were conducted, the second "AIST" satellite (RS-41at) performed 11768 circulations with 4897 communication sessions. The shortest communication session is 32 seconds, the longest one 620 seconds. The telemetry file contains 1440 measurements ( 1 measurement per minute) of 126 parameters.

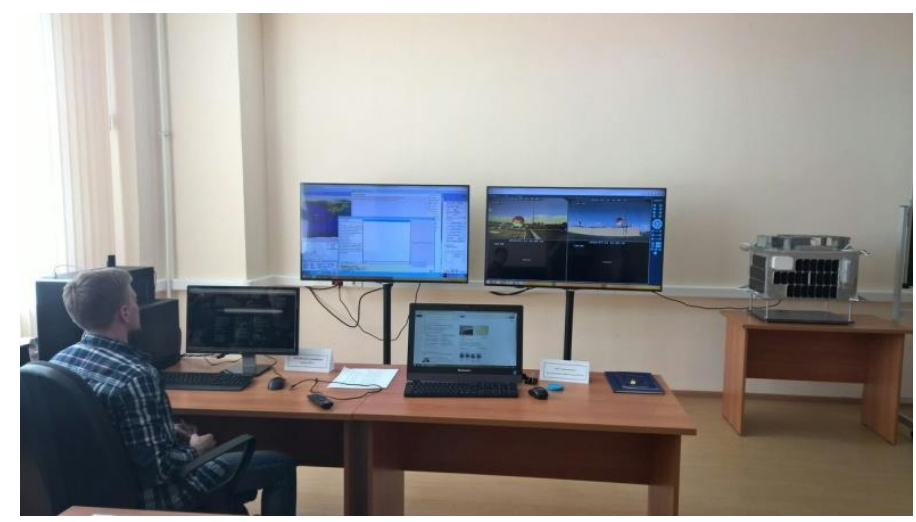

Fig. 2. - Samara University ground control center 


\subsection{Method of the research}

A comparison of panels temperatures of the satellite obtained from the telemetry and during the ground testing was conducted in order to validate the actual values of the heat flux, achieved during the ground thermal testing in the "Overheat" mode. In order to perform the comparison, the diagrams of average daily temperatures of the satellite's panels were created (Figure 3). Those diagrams show shadow parts of the satellites trajectories. The envelop curves for the shadow periods were obtained using the TLE data with the following equations [2]:

$\mathrm{T}_{\text {др }}=\frac{86400}{t_{\text {cp }}}$

where $\mathbf{T}_{д р}$ is the nodical period of the satellite; $\boldsymbol{t}_{\mathrm{cp}}$ - revolution period;

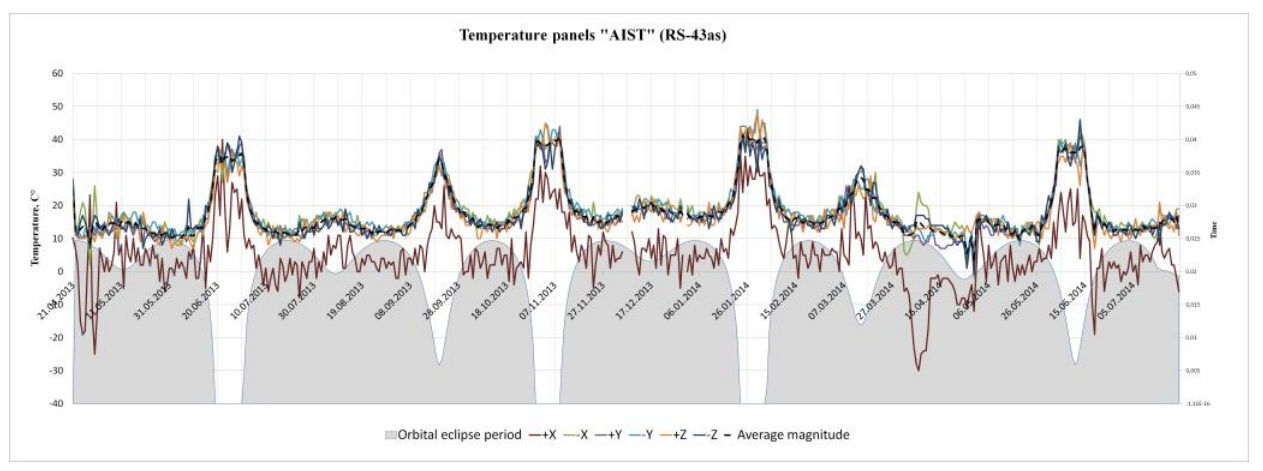

a)

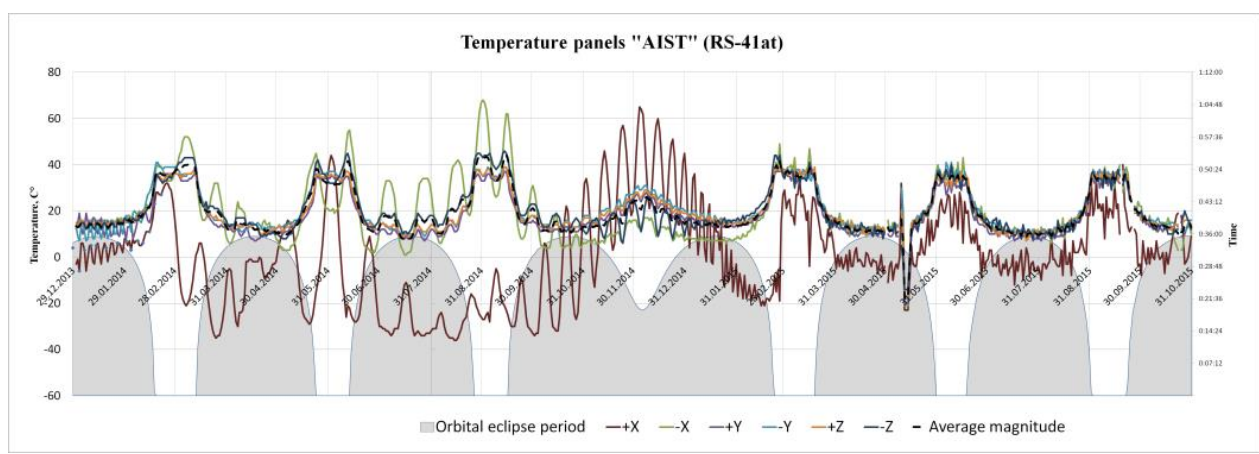

b)

Fig. 3. Graphs of the average daily temperature on the panels of satellites: a- AIST (RS-43as); b - AIST (RS-41at)

$\sin \beta^{*}=\frac{R_{3}}{R_{3}+h}$ 
where $\beta^{*}$ is the critical angle between the direction towards the Sun and the orbit plane;

R3 - is the radius of the Earth;

$\mathrm{h}-$ is the height of the orbit;

$\mathrm{m}_{\Omega \uparrow}=\Omega-\alpha_{C}+12^{h}$

where $\mathrm{m}_{\Omega \uparrow}$ is the local time of the dragon head;

$\Omega$ is the longitude of the dragon head;

$\alpha_{C}$ is the right ascension of the Sun, measured from the vernal equinox;

$12^{h}=180^{\circ}$;

$\cos \varphi_{\mathrm{T}}=\frac{\cos \beta^{*}}{\cos \beta}$

where $\varphi_{\mathrm{T}}$ is the shadow angle;

$\tau_{\mathrm{T}}=\frac{\mathrm{T}_{\text {дp }}}{2 \pi} \varphi_{T}$,

where $\tau_{\mathrm{T}}$ is the movement time in a circular orbit.

After comparison between the telemetry and the data, obtained during thermalvacuum testing we can state that there is a discrepancy between the estimated and the measured temperature of the satellite. The discrepancy is most likely to be caused by the inaccuracies of the flight conditions simulation, in particular, the heat flux magnitudes.

The inaccuracies of the ground testing could also be caused by the characteristics of the TVU400-05 testing unit that can only use infrared emission to imitate the total heat flux. In order to perform more accurate tests, the testing unit capable of more accurate simulation is needed. However, it is costly and its instalment would take a lot of time, making it inefficient. Instead, the existing testing unit needs to be upgraded with separate imitators of the Earth's radiation.

In order to verify the thermal-vacuum testing procedures, two software systems were used - ANSYS and TERM. Simultaneous use of both systems allows to work around certain disadvantages of both systems [3]. TERM was used to replicate the calculation, obtained during thermal-vacuum testing. ANSYS was utilized to estimate the thermal flows, necessary to heat up the satellite to the temperatures, obtained from TERM.

The aim of the work is to update values of design variables in the simulation of the spacecraft's operating conditions in orbit.

\section{$2 \quad$ Model characteristics}

\subsection{Satellite's model in TERM}

To calculate the temperature of structural elements we utilized TERM software, which performs calculations using the method of heat balances (also called the meth- 
od of lumped parameters or method of isothermal nodes or zones). Figure 4 shows the result of the calculation of heat flows that affect the satellite in the "Overheat" condition.
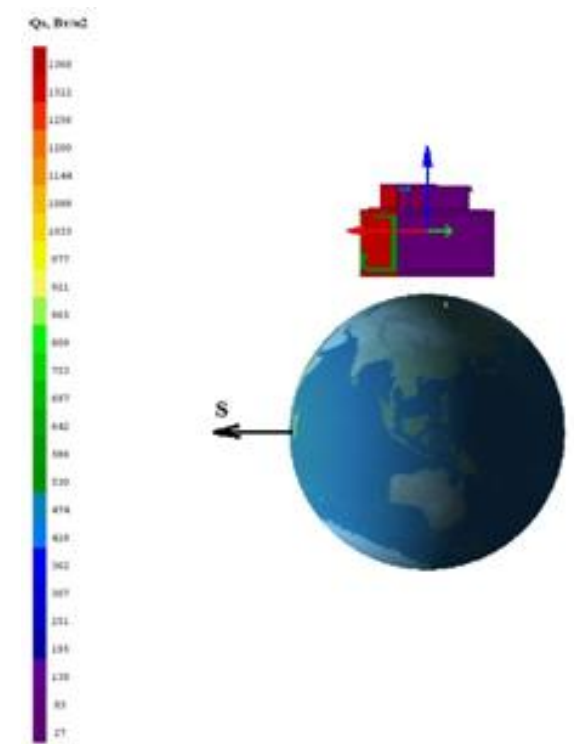

Fig. 4. Result of the calculation of heat flows that affect the "AIST" satellite in the "Overheat" condition

The heat balance method involves dissection of the structure onto L number of isothermal nodes. Each surface is given a solar radiation absorption coefficient - As, emissivity factor $-\mathrm{e}$, thermal resistance $-\mathrm{R}$ and the applied heat flux. Radiative couplings are calculated between all the surfaces. For each node, a heat balance equation is generated, forming a system of differential equations:

$\mathrm{m}_{\mathrm{i}} \mathrm{c}_{\mathrm{i}} \frac{d T_{i}}{d \tau}=\mathrm{Q}_{\mathrm{ki}}+\mathrm{Q}_{\mathrm{ni}}+\mathrm{Q}_{\mathrm{ri}}+\mathrm{Q}_{\mathrm{vi}}+\mathrm{Q}_{\mathrm{ai}}$

with the following initial conditions:

$\mathrm{T}_{\mathrm{i}}(0)=\mathrm{T}_{\mathrm{i}}^{0}, 1 \leq \mathrm{i} \leq \mathrm{L}$

where

$\mathrm{m}_{\mathrm{i}} \mathrm{c}_{\mathrm{i}}$ - mass and heat capacity of the $i$ node;

$\mathrm{T}_{\mathrm{i}}$ - temperature of the node, $\mathrm{K}$;

$\tau$ - time, s;

$\mathrm{Q}_{\mathrm{ki}}$ - conductive nodal thermal flux, $\mathrm{W}$;

$\mathrm{Q}_{\mathrm{ni}}$ - nonlinear nodal thermal flux, $\mathrm{W}$;

$\mathrm{Q}_{\mathrm{ri}}$ - resulting nodal radiative heat flux, $\mathrm{W}$;

$Q_{v i}$ - internal nodal emission flux, W;

$\mathrm{Q}_{\mathrm{ai}}$ - atmospheric nodal heat flux, W. 
Conductive nodal thermal flux is defined as:

$\mathrm{Q}_{\mathrm{ki}}=\sum_{\mathrm{k}=1}^{n} \mathrm{P}_{\mathrm{ik}}\left(\mathrm{T}_{\mathrm{k}}-\mathrm{T}_{\mathrm{i}}\right)$

where

$\mathrm{P}_{\mathrm{ik}}$ - thermal conductivity of the link between $i$ and $\mathrm{k}$ nodes, W/K;

$\mathrm{T}_{\mathrm{k}}$ - temperature of the $\mathrm{k}$ node, that is connected to the $i$ node with a thermal link $\mathrm{P}_{\mathrm{ik}}$, $\mathrm{K}$

$\mathrm{n}$ - the amount of heat links of the conductive heat links of the $i$ node.

Nonlinear heat flux $\mathrm{Q}_{\mathrm{ni}}$ towards the $i$ node is defined as:

$\mathrm{Q}_{\mathrm{ni}}=\sum_{\mathrm{k}=1}^{\mathrm{n}} \mathrm{A}_{\mathrm{ik}}\left(\mathrm{T}_{\mathrm{k}}^{4}-\mathrm{T}_{\mathrm{i}}^{4}\right)$

where

$\mathrm{A}_{\mathrm{ik}}$ - the radiative heat exchange between the $i$ and k nodes;

$\mathrm{T}_{\mathrm{k}}$ - temperature of the $\mathrm{k}$ node, that is connected to the $i$ node with a thermal link $\mathrm{A}_{\mathrm{ik}}$, $\mathrm{K}$;

$\mathrm{n}$ - the amount of nonlinear heat links of the $i$ node.

Resulting nodal heat flux $Q_{\mathrm{ri}}$ is defined as:

$Q_{\mathrm{ri}}=\sum_{\mathrm{j}=1}^{\mathrm{n}} \mathrm{F}_{\mathrm{j}}\left(\mathrm{q}_{\mathrm{ai}}-\varepsilon_{\mathrm{j}} \sigma \mathrm{T}_{\mathrm{j}}^{4}\right)$

where

$\mathrm{n}-$ the amount of surfaces, belonging to the $i$ node;

$\mathrm{F}_{\mathrm{j}-}$ the area of the $\mathrm{j}$ surface, belonging to the $i$ node, $\mathrm{m}^{2}$;

$\mathrm{q}_{\mathrm{aj}}$; - radiant flux density, absorbed by the $j$ surface, $\mathrm{W} / \mathrm{m}^{2}$;

$\varepsilon_{\mathrm{j}} \mathrm{T}_{\mathrm{j}}$ - emissivity coefficient and the $j$ surface temperature;

Atmospheric nodal heat flux is defined as:

$Q_{a i}=\sum F_{j}\left(q_{m j}+q_{r j}\right)$

where

$\mathrm{q}_{\mathrm{mj}}, \mathrm{q}_{\mathrm{rj}}-$ molecular and recombination flux.

Internal nodal heat emissions are defined in a form of sequence diagrams - values of $Q_{\mathrm{vi}}\left(\tau_{0}\right), Q_{\mathrm{vi}}\left(\tau_{1}\right) \ldots \mathrm{Q}_{\mathrm{vi}}\left(\tau_{\mathrm{q}}\right)$ in times $\tau_{0}, \tau_{1}, \ldots \tau_{\mathrm{q}}$. The $\mathrm{Q}_{\mathrm{vi}}$ is approximated based on those values as a linear or a lattice time function. On the time interval $\tau_{\mathrm{q}-1} \leq \tau \leq \tau_{\mathrm{q}}$ $Q_{v i}$ is either constant $Q_{v i}\left(\tau_{q-1}\right)$, or linearly changes its value form $Q_{v i}\left(\tau_{q-1}\right)$ to $\mathrm{Q}_{\mathrm{vi}}\left(\tau_{\mathrm{q}}\right)$.

The nodal temperatures are obtained as a result of solving the above mentioned system of equations. The temperature fields of the required level of fidelity can be obtained by alteration of the amount of nodes.

The values of the heat flows and panel temperatures, calculated in TERM, are similar to those that were measures during thermal-vacuum tests, however, they diverge with the results of telemetry analysis, which gives grounds to state that the resulting heat flux was underestimated during the testing. 


\subsection{ANSYS thermal model}

The purpose of the thermal analysis is the mathematical modeling of heat flows affecting the AIST small satellite in the "Overheat" mode. External heat sources are the Sun and the Earth. In the "Overheat" mode the main heat flux comes from the Sun and the heat flux from the Earth does not exceed 5\% of the solar flux. The resulting heat flux was set to $1460 \mathrm{~W} / \mathrm{m}^{2}$.

The initial temperature of the satellite was set to be $40^{\circ} \mathrm{C}$, as the averaged measured value, obtained from telemetry [4].

The analysis was carried out for the following starting conditions:

- inclination of the satellite's orbit is $90^{\circ}$;

- initial temperature for all structural elements is $40^{\circ} \mathrm{C}$;

- satellite stays in the "Overheat" mode for 24 hours;

- the heat flux during "Overheat" mode is constant;

- the satellite is made from composite aluminum panels, covered with photo elements made of GaAs.

The value of the total heat flux on the satellite was used as a variable parameter in the calculation.

A simplified three-dimensional model of the ICA was developed for calculation in the ANSYS software package. Figure 5 shows the calculated temperature values. The result of the calculation of the satellite's surface temperatures at the heat flux corresponding to the obtained in the TERM program is close to the results of the spacecraft ground tests, but differs from the device operating temperatures, obtained from telemetry data.

In order to determine the actual heat flux we solved an inverse problem, increasing the heat flux magnitude until we match the temperature values, obtained from telemetry data (which is approximately $60^{\circ}$ ). Figure 6 shows temperature distribution for corrected heat flux. Thus we come to the conclusion that increase in the value of specific heat flux on the panel $+\mathrm{Y}$ from $1400 \mathrm{~W} / \mathrm{m}^{2}$ to $2275 \mathrm{~W} / \mathrm{m}^{2}$ gives values of surface temperatures close to the ones obtained from telemetry data, which suggests that the magnitude of the heat flux during the test in "Overheat 2" mode was underestimated compared to the real one.

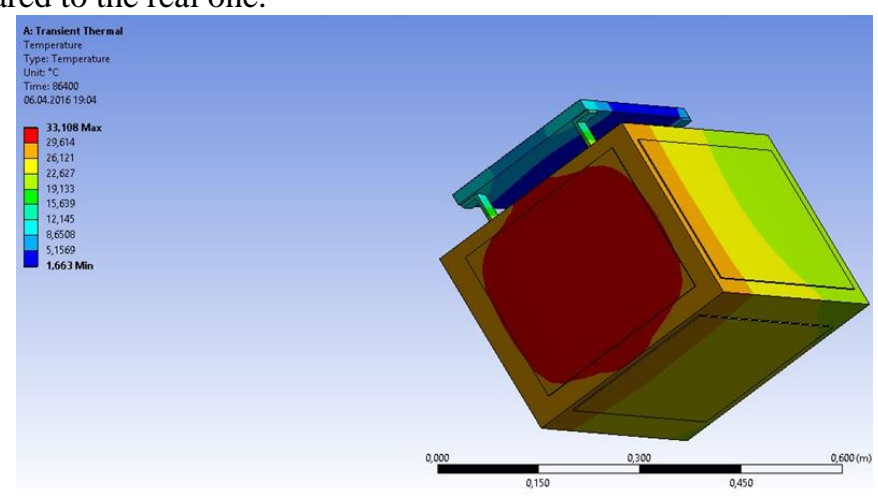

Fig. 5. Temperature values for the initial heat flux magnitude 


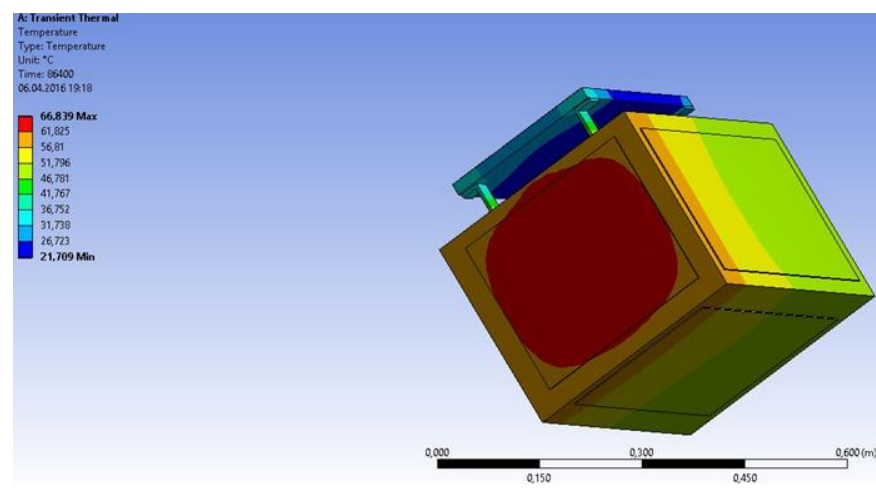

Fig. 6. Temperature values for the corrected heat flux magnitude

\section{Conclusion}

The concluded study on verification of the ground testing parameters for the thermal control system of the AIST small satellites based on the telemetry data, obtained using SSAU ground control center, allows to state that the initial conditions for the thermal-vacuum testing, in particular the heat flux magnitude, need to be corrected. Based on the mathematical modeling performed in "TERM" and "ANSYS" software packages a corrected value of the heat flow to the satellite in the "Overheat" mode was suggested.

The results of the study confirms the feasibility and effectiveness of using satellite flight operation data for the enhancement of ground testing and improving the reliability of new on onboard systems and their prototypes.

\section{References}

1. Tkachenko SI, Salmin VV, Syomkin ND, Kurenkov VI, Abrashkin VI, Prokhorov AG, Safronov SL, Tkachenko IS, Petrukhina KV. The design and main characteristics of small SSAU spacecraft - RCC Progress "Samara Space Center". Vestnik SSAU, 2010; 2: 154165. [In Russian]

2. Chernov AA, Cherniavsky GM. The orbits of the Earth remote sensing satellites. Moscow: Lektsii i uprazhneniya, "Radio and Communications" publ., 2004; 200 p. [In Russian]

3. Kulikov DS, Shatin AA, Vel'misov IG. Automated creation structural elements of the spacecraft thermal model. Vestnik SSAU, 2013; 41(3): 139. [In Russian]

4. Tkachenko SI, Salmin VV, Tkachenko IS, Safronov SL, Kaurov IV, Ivanushkin MA, Volgin SS, Korovin MD. "AIST" small satellites' family, a joint project between RSC "Progress" and SSAU, thermocontrol system performance analysis. International Conference on Mechanical Engineering, Automation and Control Systems (MEACS), 1-4 Dec., 2015. 